\title{
RECYCLING OF HYDROGEN ISOTOPES- FROM AN IDENTIFICATION OF MECHANISMS IN TEXTOR TO A WIDER FORMULATION
}

\author{
PH. MERTENS* and S. BREZINSEK Institut für Plasmaphysik, Forschungszentrum Jülich GmbH \\ Euratom Association, Trilateral Euregio Cluster, D-52425 Jülich, Germany
}

Received June 4, 2004

Accepted for Publication October 8, 2004

The detailed physical mechanisms of hydrogen recycling are not yet completely clear. But, their understanding is required for the correct interpretation of spectroscopic measurements that are intended to provide us routinely with the total particle fluxes as well as with sound extrapolations to fusion devices of the next generation. Thanks to its large observation ports, TEXTOR provides ideal conditions for the combination of optical diagnostics based on completely different techniques, which can be applied simultaneously, with high resolving powers $\left(\lambda / \Delta \lambda=2 \times 10^{4}\right.$ to $\left.2 \times 10^{5}\right)$.

It is shown how Zeeman spectroscopy on the Balmeralpha transition ( $\lambda 656.1 \mathrm{~nm}$ ) and laser-induced fluorescence at Lyman-alpha ( $\lambda 121.5 \mathrm{~nm})$ both point to the presence of a substantial amount of cold hydrogen atoms (with kinetic energy $<1 \mathrm{eV}$ ) in front of plasma-facing components, which is a phenomenon that, surprisingly, is largely independent of the local plasma parameters. This has led to a strong development of the spectroscopy of hydrogen molecules (Fulcher band), which may be a dominant source of atomic hydrogen in the plasma edge, and, as a final result, to an explanation for the phenomenological correction applied to the inverse photon efficiencies $S / X B$ that are commonly used in the conversion of the photon fluxes into particle fluxes.

KEYWORDS: hydrogen recycling, laser-induced fluorescence, plasma-wall interaction

\section{INTRODUCTION: THE BASICS OF RECYCLING}

Hydrogen and deuterium are the fuel of present-day tokamaks. It is still a rather complex task, though, to

\footnotetext{
*E-mail: Ph.Mertens@fz-juelich.de
}

determine how neutrals and ions of these isotopes behave in the vicinity of the main chamber wall or in front of plasma-facing components like limiters or divertor plates. On one hand, a steady particle flux of atoms and ions, which escape the core plasma owing to imperfect confinement, impinges onto the exposed surfaces; on the other hand, the same particles are eventually released back into the plasma after neutralization, building in turn a comparable inward flux. This process is called recycling. The recycling coefficient is given by

$$
R=\frac{\text { particle flux from the wall }}{\text { particle flux onto the wall }} .
$$

The order of magnitude of the recycling fluxes $(R \approx 1$ for a coarse estimate), which obviously depend on the plasma parameters but also depend very much on the considered area in case of limiters, may be given for typical discharges in TEXTOR (Refs. 1 and 2). The total particle fluxes on the second line are only indicative and correspond to appropriate mean values of $\Gamma$ on the respective first line, hence the "larger than/equal" signs:

limiter:

$$
\begin{aligned}
& \Gamma_{\mathrm{D}, \text { lim }} \approx 10^{21} \text { to } 10^{24} \mathrm{~m}^{-2} \cdot \mathrm{s}^{-1}, \\
& \Phi_{\text {Lim }} \geq \int \Gamma d A=\mathcal{O}\left(6 \times 10^{21}\right) \mathrm{s}^{-1},
\end{aligned}
$$

wall:

$$
\begin{aligned}
\Gamma_{\mathrm{D}, \text { wall }} & \approx 10^{19} \text { to } 10^{20} \mathrm{~m}^{-2} \cdot \mathrm{s}^{-1}, \\
\Phi_{\text {Wall }} & \geq \int \Gamma d A=\mathcal{O}\left(1.5 \times 10^{21}\right) \mathrm{s}^{-1} .
\end{aligned}
$$

Taking the particle confinement time into consideration, $\tau_{p} \approx \mathcal{O}(60 \mathrm{~ms})$, and the flat-top plasma duration of the order of $6 \mathrm{~s}$, one sees in a rough estimation (neglecting net pumping and external fueling) that a particle undergoes the recycling process about 100 times on average during the plasma pulse. 
It is clear that deviations from $R=1$ cannot last forever for the intended, steady-state plasmas, although hydrogen pumping by the walls can take place without interruption over long discharges as shown in different fusion devices, for instance, in Refs. 3, 4, and 5. Figure 1a shows a tangential view of a poloidal cross section of the tokamak in Balmer-alpha light during a plasma discharge. This is the light that is emitted by hydrogen atoms upon excitation by electron impact to their $n=3$ level, where $n$ denotes the principal quantum number. The corresponding recycling process on the toroidal belt limiter Advanced Limiter Test (ALT-II) is depicted schematically in Fig. 1b.

As a matter of fact, the ratio of ions to atoms in the incident flux is not known accurately a priori; also not known are the ratio of atoms to molecules in the flux of released particles and whether this release corresponds to bare reflection or, for instance, to desorption after
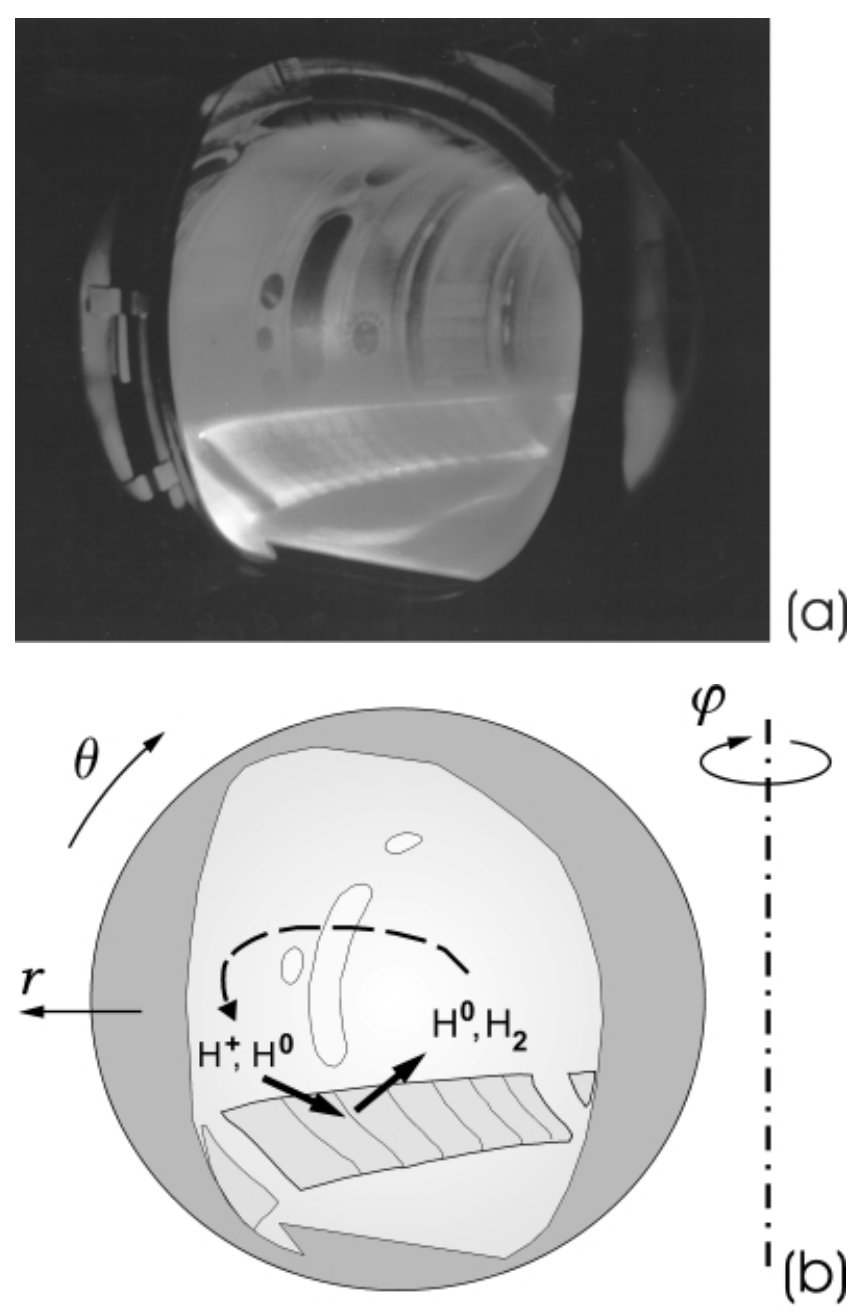

Fig. 1. Recycling at the toroidal belt limiter in TEXTOR: (a) live picture of the poloidal cross section in Balmeralpha light and (b) schematics of the recycling process. recombination. The physical mechanisms underlying the recycling process are not yet completely identified.

Important quantities that have to be measured to elucidate the question are, among others, the spatially resolved atomic density $n_{\mathrm{D} 0}$ and velocity distributions $f(v)$ in front of the plasma-facing components, i.e.,

$$
n_{\mathrm{D} 0}(r ; \theta, \varphi) \text { and } f_{\mathrm{D} 0}(v, r, \theta, \varphi)
$$

at the radius $r$, with $\theta$ and $\varphi$ the poloidal and toroidal angles, respectively.

Derived values are the penetration depth $\ell \sim\langle v\rangle /$ $\left(n_{e}\langle\sigma v\rangle_{i o n}\right)$, where $n_{e}$ stands for the local electron density and $\langle\sigma v\rangle_{i o n}$ stands for the rate coefficient for electron impact ionization, and the flux $\Gamma_{\mathrm{D} 0}$ (for a discussion of $\ell$, see Ref. 6). Note that deuterium will be used in the following as representative for all hydrogen isotopes, from hydrogen to tritium [as the case arises, for instance, in the Joint European Torus (JET) tokamak]; hence, the $\mathrm{D}^{0}$ subscripts denote atomic deuterium in the foregoing formulas. The importance of recycling effects is stressed in the recent literature, for instance, in Refs. 7, 8, and 9 and detailed processes in Ref. 10.

\section{LIF IN THE VUV: A NOVEL EXPERIMENTAL SETUP}

With the aim of complementing the techniques of emission spectroscopy, which owing to signal integration along the line of sight miss the spatial resolution, a dedicated laser-induced fluorescence (LIF) setup was developed and installed on TEXTOR (Refs. 11 and 12). This system takes advantage of the high accessibility of the different ports, a remarkable feature of the tokamak in Jülich that is especially desirable for LIF: Whereas the excitation by laser light takes place selectively in the direction of the laser beam, the subsequent spontaneous emission of fluorescence light is distributed over the full solid angle of $4 \pi$ (steradians). Clearly, one has to make a point of collecting as much light as possible. The general LIF setup on TEXTOR is described elsewhere in the present volume, especially as far as it is used for measurements at a limiter lock. Similar fluorescence measurements carried out on former fusion devices, albeit at the Balmer-alpha wavelength, ought to be mentioned, such as Refs. 13 and 14. An overview of the different laser techniques is given in Ref. 15.

The case of deuterium implies a sophisticated arrangement when the laser is supposed to excite atoms from the ground state (see below, Fig. 8 on p. 166, the Grotrian diagram of the hydrogen atom). This can be done with a two-photon scheme, as demonstrated earlier on Heliotron E (Refs. 16 and 17), or by means of singlephoton excitation as performed on TEXTOR.

According to this requirement, the laser system delivers $L_{\alpha}$ radiation (Lyman-alpha) at the wavelength of $\lambda=121.5 \mathrm{~nm}$, i.e., in the vacuum-ultraviolet (VUV) 


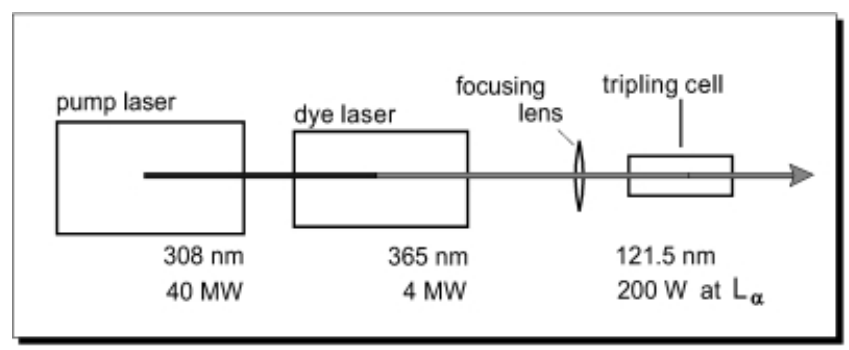

Fig. 2. Schema of the three-step laser system for production of VUV radiation at $L_{\alpha}$.

spectral range. Such radiation in the VUV range is not within the reach of commercially available lasers. The system that was developed for this particular goal is based on an excimer-pumped dye laser that produces at least $50 \mathrm{~mJ} /$ pulse $\left(\Delta t_{L A S} \approx 15 \mathrm{~ns}\right)$ at a typical repetition rate of 20 to $50 \mathrm{~Hz}$ - the higher the better as far as time resolution or statistics is concerned. The wavelength lies in the near-ultraviolet range around $\lambda=365 \mathrm{~nm}$. The recently improved unit relies on a double-grating arrangement to ensure a narrow spectral width. The frequency of this laser radiation is tripled in a mixture of krypton and argon, ${ }^{18,19}$ in a noble gas cell, giving routinely $>200 \mathrm{~W}$ for $\sim 5 \mathrm{~ns}$ (Ref. 20). The three units used are sketched in Fig. 2. Thanks to the narrow spectral width of $\Delta \lambda_{L A S} \approx$ $0.6 \mathrm{pm}$ in the VUV range, a so-called resolving power of $\mathcal{R} \equiv \lambda / \Delta \lambda \geq 200000$ is obtained without the need for inserting and adjusting any additional optical com- ponent, such as an intracavity Fabry-Pérot étalon or the like. ${ }^{21}$ This simply means that the VUV radiation that is available permits the recording of the velocity distributions of even the slowest atoms, as will be shown below.

The setup would be useless without an observation system complying with both of the requirements previously stated, namely, the collection of far-ultraviolet light (i) within an appreciable solid angle (ii). The complete experimental setup is shown in Fig. 3. Two cassegrainian telescopes image the fluorescence volume (from top, $\sim 1 \mathrm{~cm}^{3}$ ) onto synchronously gated, solar-blind photomultipliers. The main mirror of the upper one can be tilted back and forth to scan radial profiles along the laser beam path. ${ }^{12}$ The other much more compact observation system (see diagnostics section) is equally suited to LIF measurements at $L_{\alpha}$, at some expense of the signal-tonoise ratio, which is only noticeable in the far wings of the fluorescence profiles. It is installed in the section of the torus where a vacuum lock permits insertion of various plasma-facing components from the bottom. LIF measurements are thus performed in front of limiter heads as well, with an enhanced radial resolution, down to $3 \mathrm{~mm}$ (Ref. 22).

Finally, it should be stressed that thanks to the spectrally narrow, selective excitation, the velocity distributions of atomic deuterium can be measured according to the Doppler relation $v=\left[\left(\lambda-\lambda_{0}\right) c\right] / \lambda_{0}$, where the reference wavelength $\lambda_{0}$ corresponds to the laser tuned to the transition of atoms at rest. The geometry displayed in Fig. 4 illustrates how a red shift in the selective excitation corresponds to atoms moving against the laser beam

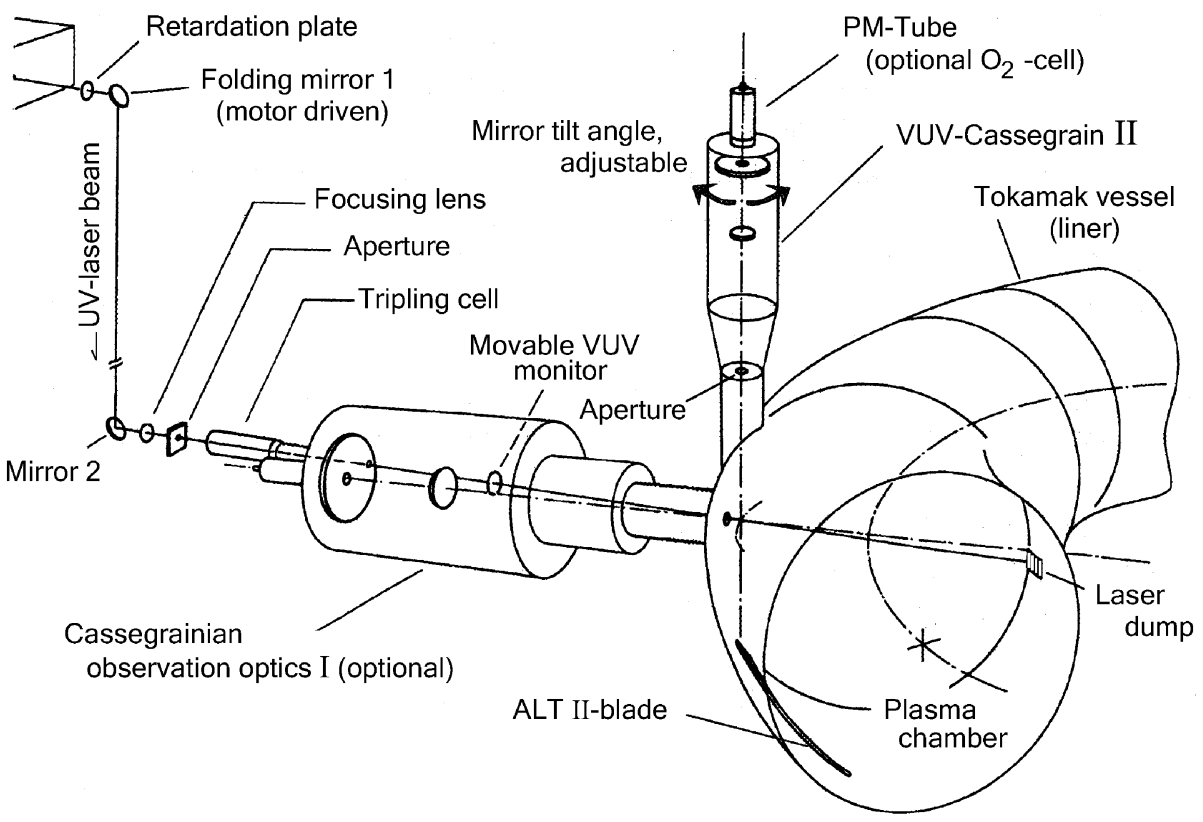

Fig. 3. Experimental setup for LIF at $L_{\alpha}(\lambda 121.534 \mathrm{~nm})$ in front of the first wall of TEXTOR (liner); see Ref. 12. The corresponding arrangement at the test limiter is presented in the diagnostics section. 


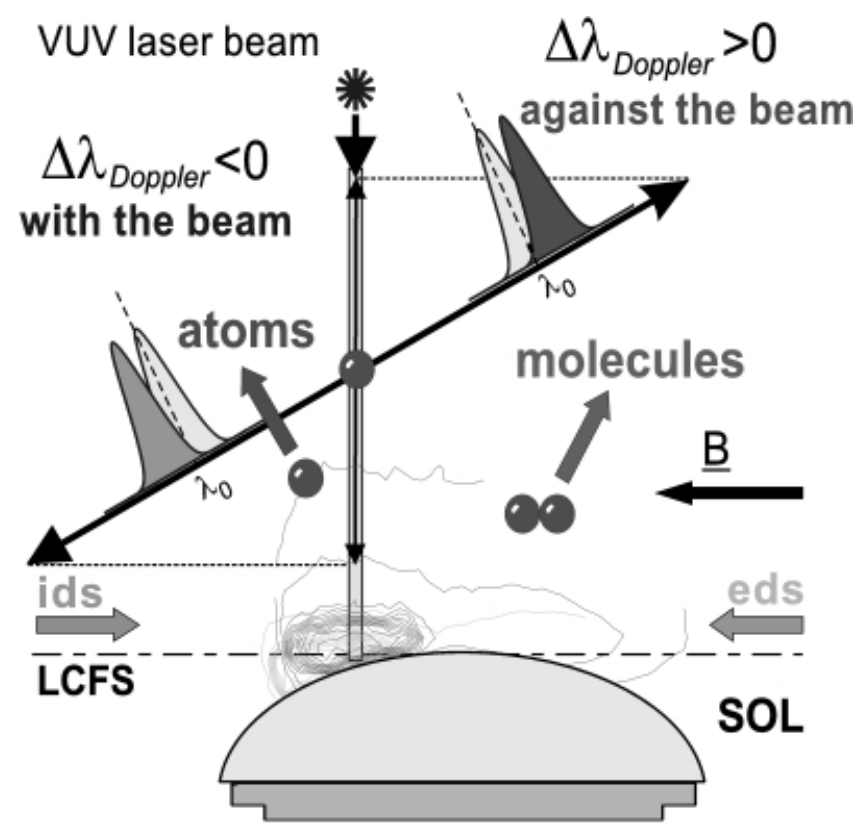

Fig. 4. Bare geometry with respective directions of particles and laser beam (the plasma-facing component shown is a mushroom limiter).

(escaping the surface), whereas those that are moving along with the beam, i.e., toward the plasma-facing surface, exhibit a blue wavelength shift.

A high signal-to-noise ratio could be obtained in the center of the spectral line with the largest, horizontal telescope seen in the equatorial plane of the tokamak (Fig. 3). Early examples of raw fluorescence signals with $S / N=30$ to 40 are shown in Fig. 5 for two laser pulses in

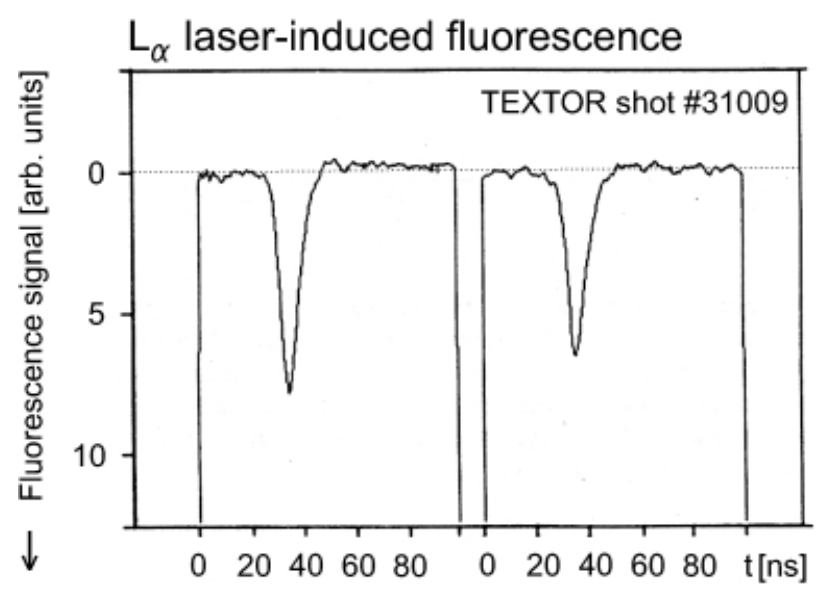

Fig. 5. Two fluorescence pulses (photomultiplier raw signals, downward) with high $S / N$ ratio (see Ref. 11). sequence. ${ }^{11}$ Although all other compact telescopes naturally tend to degrade the signal quality, values of 5 to 10 can still be obtained at the other locations around the torus.

\section{LIF: RADIAL AND SPECTRAL PROFILES}

While the radial profiles looked about as expected (Fig. 6a, where the solid curve is just to guide the eye, and Ref. 12), with a steep decay that starts around the last closed flux surface (LCFS), spectral profiles, that is, those mapping the atomic velocity distributions, revealed extremely low wavelength shifts, $\sim 4$ pm, i.e., velocities $\sim 1 \times 10^{4} \mathrm{~m} / \mathrm{s}$ or (in energy units) $1 \mathrm{eV}$, respectively. ${ }^{11,12,22}$ This was unexpected, ${ }^{\mathrm{a}}$ as the same picture was recorded equally in front of the TEXTOR liner or in front of a limiter, i.e., at and within the LCFS, where electron temperatures lie in a broad range between 20 and $100 \mathrm{eV}$. Moreover, it is almost independent of the type of discharge and of the plasma parameters. A few profiles are shown in Fig. 7. They even display different structures, the discussion of which is outside the scope of the present paper. It may be pointed out, though, that these are intricately connected both to the temperature of the limiter surface (see Sec. IV) and to possible collisions of the measured deuterium atoms with the bath of hot deuterons ( $\sim 100 \mathrm{eV}$, e.g., charge-exchange particles) escaping the plasma.

Noteworthy, again, is that all profiles present a substantial contribution below the $1-\mathrm{eV}$ limit stressed by the vertical bar. Other cases are discussed in Refs. 12, 22, and 25 , as far as spectral profiles and their implications for the penetration depths are concerned. Reference 12 deals exclusively with spatial profiles recorded with LIF at $L_{\alpha}$ in the vicinity of the first wall, the so-called liner of TEXTOR, whereas Ref. 22 presents results in front of a limiter head. LIF at $L_{\alpha}$ can so far measure atomic deuterium shifts up to $17 \mathrm{pm}$, i.e., below $\sim 19 \mathrm{eV}$, depending on the local density but definitely not charge-exchange neutrals that are expected at much higher energies. This corresponds to a detection limit of $\sim 5 \times 10^{14} \mathrm{~m}^{-3}$ in terms of densities. ${ }^{12,26}$

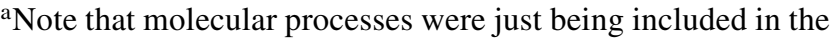
numerical codes at that time, partly on our impulse and as a result of these very measurements. This explains that the substantial amount of "slow" atoms, which is satisfactorily accounted for in the present case, may have been in discrepancy for conditions different from wall vicinity. EIRENE will be re-run in the future to produce velocity distributions comparable to the LIF measurement range, keeping in mind that - the flux being considered equal-low velocities are "overrepresented" in the fluorescence signal owing to their higher densities (see also Ref. 23 where some new simulations can be found).
} 


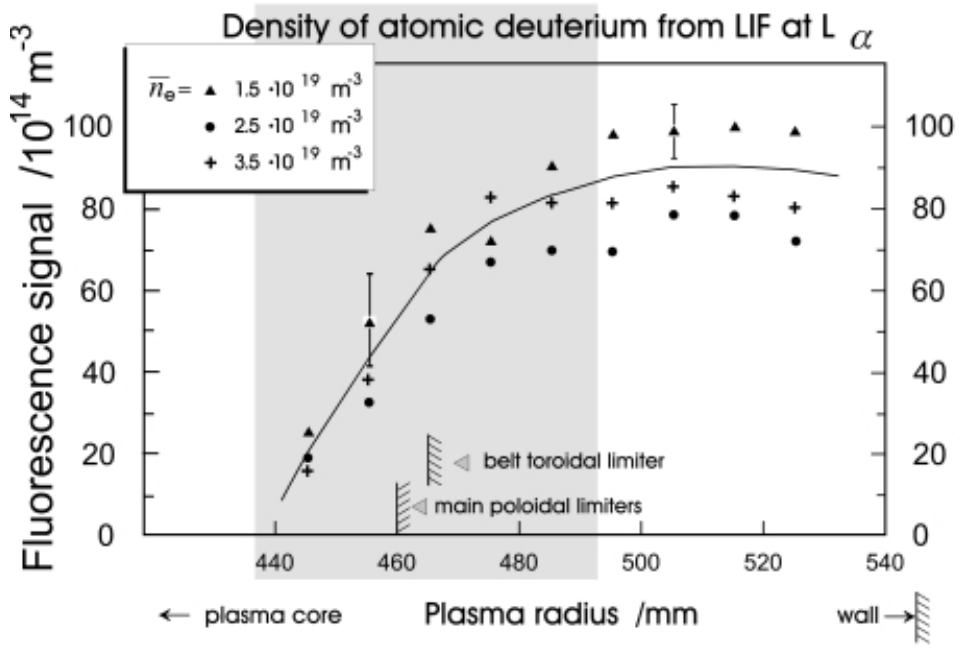

(a)

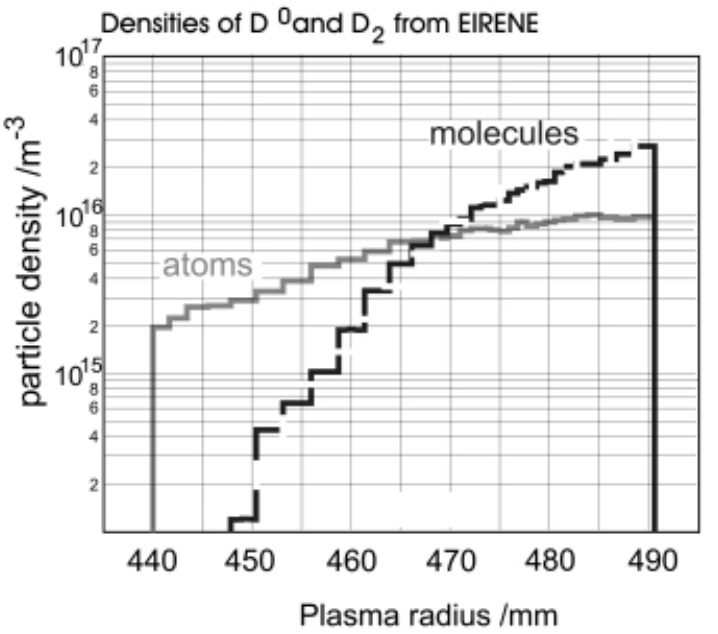

(b)

Fig. 6. An EIRENE (see Ref. 23 for details on the EIRENE computer code) simulation of the atomic, radial density profiles in front of the wall of the plasma chamber ( $\mathrm{D}^{0}$, Fig. $6 \mathrm{~b}$, solid curve marked "atoms" for $\bar{n}_{e}=1.5 \times 10^{19} \mathrm{~m}^{-3}$ ) is in reasonable agreement with the atomic densities measured with LIF (Fig. 6a, triangles). The simulated area is shaded in gray. Molecular processes were just being implemented, after their importance had been duly recognized (see footnote a on p. 164). Exact geometry and position of the region of interest in the poloidal cross section of the tokamak are given in Ref. 25 .

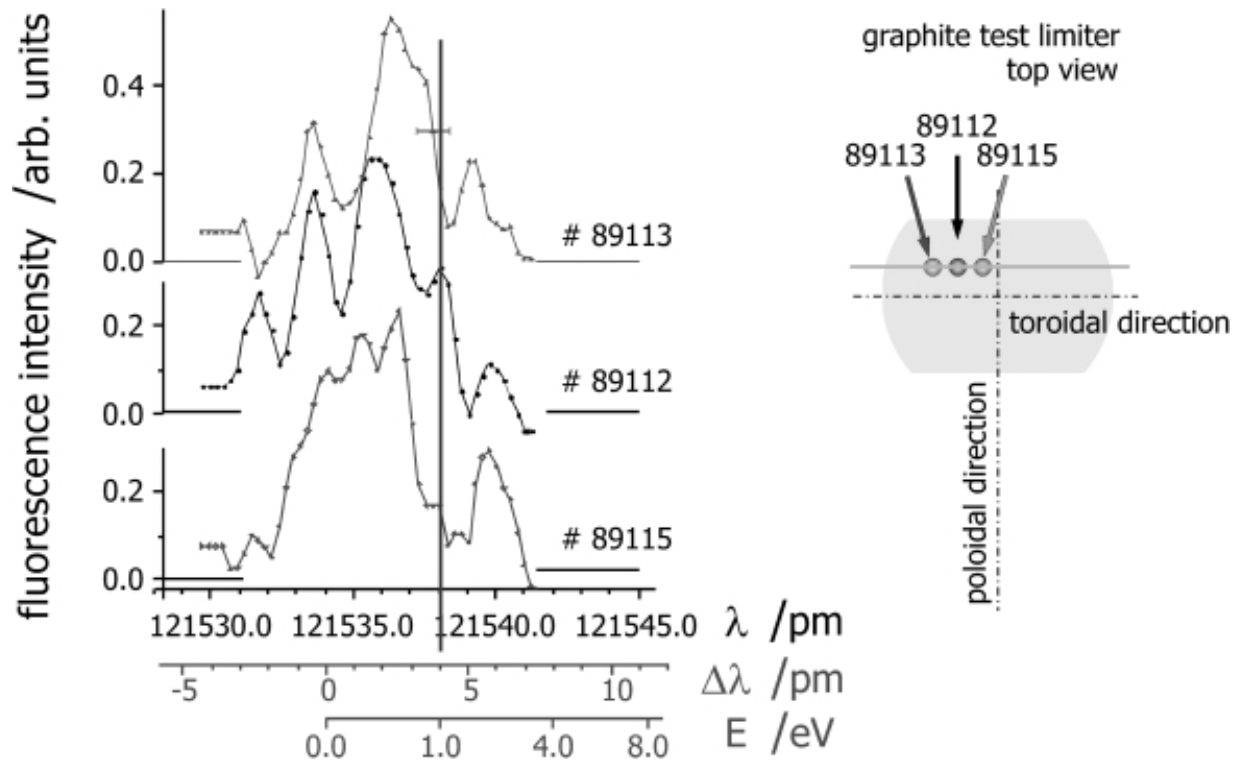

Fig. 7. Velocity distributions of atomic deuterium in the plasma edge (here in front of a graphite element; see Ref. 25). Spectral profiles at different locations have a common feature: They correspond to very low energies. The vertical bar indicates the 1-eV limit. Note that apparent velocities just below zero are mostly due to the finite laser spectral width and to a lesser extent to the uncertainty in the laser wavelength scale, which can only be cross-checked against the fluorescence of thermalized atoms in the laboratory. ${ }^{26}$

\section{EMISSION SPECTROSCOPY IN THE VISIBLE RANGE: POSSIBLE EXPLANATIONS}

At the same time, the Balmer-alpha radiation at $656 \mathrm{~nm}$ (see Fig. 8) was also scrutinized in TEXTOR, with the help of a high-resolution spectrometer- $\mathcal{R} \approx$ 40000 - around the same radius and at only slightly different poloidal locations. ${ }^{27}$ The tangential direction of observation was along the magnetic field lines. Thanks to this configuration, only the $\sigma$ components were showing 

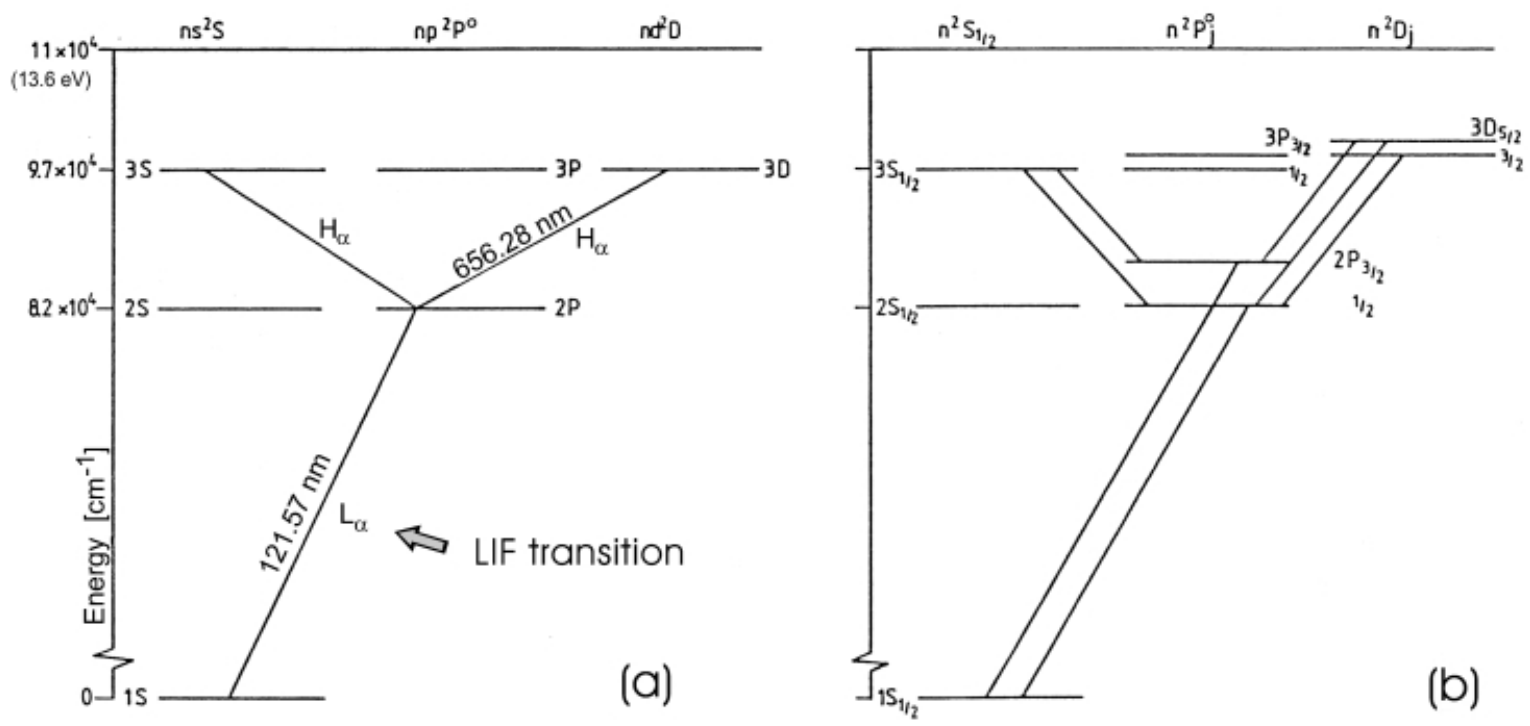

Fig. 8. Energy levels of the hydrogen atom and corresponding transitions (a) without consideration of the fine structure and (b) with consideration of the fine structure. The laser developed for TEXTOR excites the $L_{\alpha}$ transition $(n=1$ to $n=2$ at $121.5 \mathrm{~nm})$

in the well-known spectral pattern that is due to the splitting of optical components in a strong magnetic field (Zeeman and Paschen-Back effects). ${ }^{28}$ The deepness of the dip at the position of the missing $\pi$ component was surprising, though; it was another indication of the presence of a substantial amount of extremely cold (slow) atoms, to which a temperature $\sim 0.3 \mathrm{eV}$ was assigned (Fig. 9) (Ref. 27). Incidentally, such occurrences are also observed in other fusion devices ${ }^{25}$ like Tore Supra $^{29}$ or Alcator. $^{24}$

These findings were gained from two independent spectroscopic diagnostics with different techniques and geometries, which makes them indisputable. This was beyond doubt a strong advantage of combining LIF and emission spectroscopy for the detection of one particular species (moreover, additional information comes from the fact that the use of two methods allows both the ground state and higher lying ones to be sampled).

An explanation of these concordant results is given by the source of atomic deuterium in the form of molecular dissociation processes..$^{10,25}$ Potential curves for the hydrogen molecule help as a support for discussion. Whereas the products of molecular dissociation along the lowest possible repulsive curve shown in Fig. 10 (channel 1) are left with a total energy of $\sim 4.4 \mathrm{eV}$, or $\sim 2.2 \mathrm{eV} /$ atom, other paths that follow upper curves of the excited electronic states leave energies below $1 \mathrm{eV}$ after the dissociation has taken place (channel 2). This also means that the detected, slow atoms can be found mainly in $n \ell$ states with $n=1$ (ground state, detectable with LIF excitation) or, for instance, $n=3$ (the excited state that decays with concurrent emission of the ob- served Balmer radiation), hence the name of dissociative excitation that is used throughout literature. ${ }^{30}$ The threshold for such dissociation processes, along the upper repulsive curves in the diagram, lies at $17 \mathrm{eV}$ (Ref. 31).

A more accurate description with further details can be found in Ref. 32 where it is also shown that the molecular ion $\mathrm{H}_{2}^{+} / \mathrm{D}_{2}^{+}$plays a major role in specific cases. Among other effects linked to the presence of molecules is the other, at first surprising, finding that a higher local electron temperature often results in the production of colder atoms since electrons in the range of, say, $20 \mathrm{eV}$ and above are able to bring the molecules up to the involved excited states mentioned in the previous discussion. To complicate the picture, these atoms can nevertheless be heated up in the surrounding bath of hot deuterons. ${ }^{31,33}$ Moreover, the surface temperature of the plasma-facing components, from which deuterium is released, determines to a large extent the ratio of molecules to atoms in the total flux. ${ }^{34}$ Detailed discussion can be found in Refs. 25, 35, and 36 and in Sec. V.

\section{SPECTROSCOPY OF HYDROGEN MOLECULES: AN INDISPENSABLE ADDITION}

As indicated in Sec. IV, hydrogen molecules have been identified as important quantities involved in the hydrogen recycling process at plasma-facing surfaces made of graphite. The quantification of these molecules and of their contribution to the total flux of released hydrogen particles has, therefore, been an important subject in the plasma-wall research activities in Jülich. 


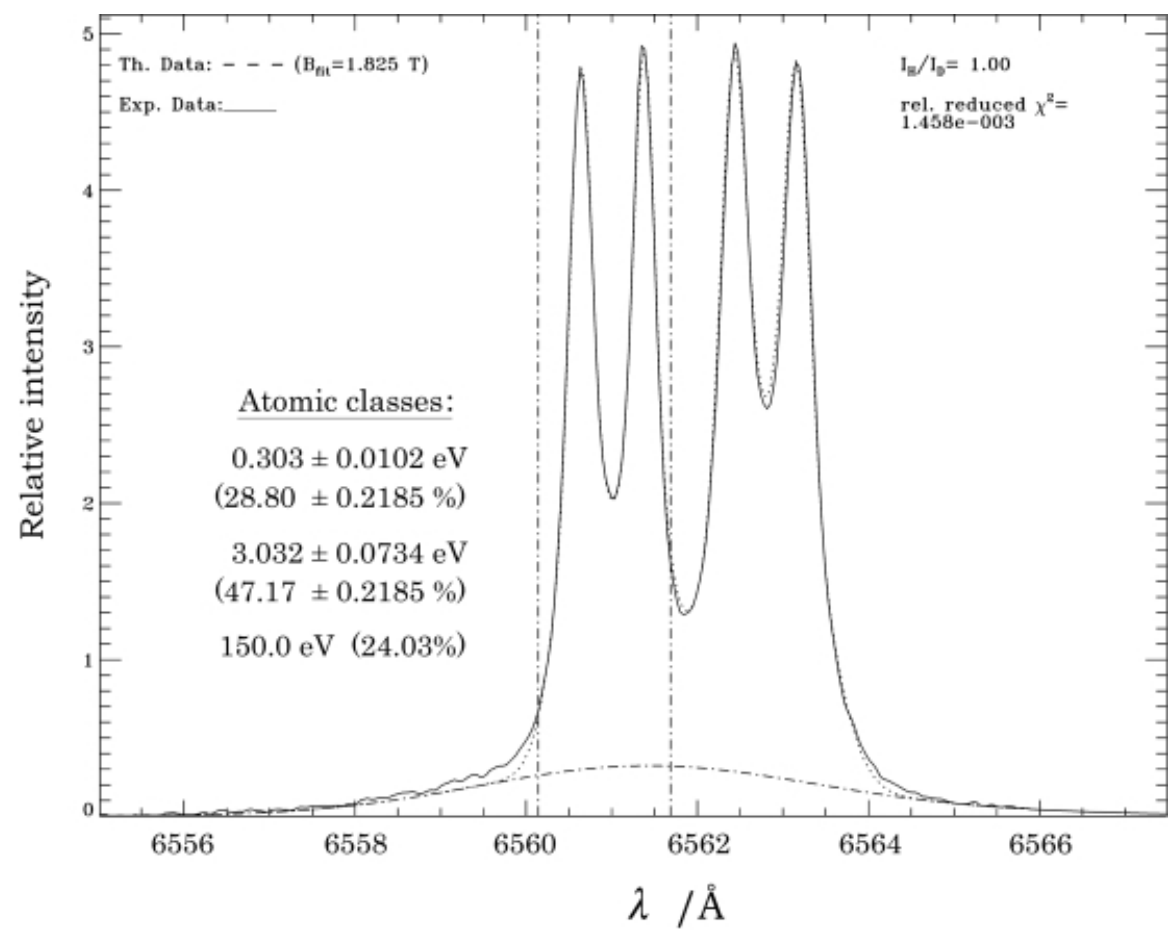

Fig. 9. Balmer spectrum of both atomic deuterium $\left(\mathrm{D}_{\alpha}\right.$, left two peaks) and hydrogen $\left(\mathrm{H}_{\alpha}\right.$, right peaks) at a plasma radius of $43.8 \mathrm{~cm}$. A magnetic field strength of $1.825 \mathrm{~T}$ was assumed for the theoretical curves (dashed/dashed-dotted). The fit corresponds to three atomic temperatures of $0.30,3.03$, and $150.0 \mathrm{eV}$ as documented in the left part of the figure with exact values and proportions. The vertical, dashed-dotted lines indicate the spectral domain covered in the fitting procedure of the low-energy components (from Ref. 27).

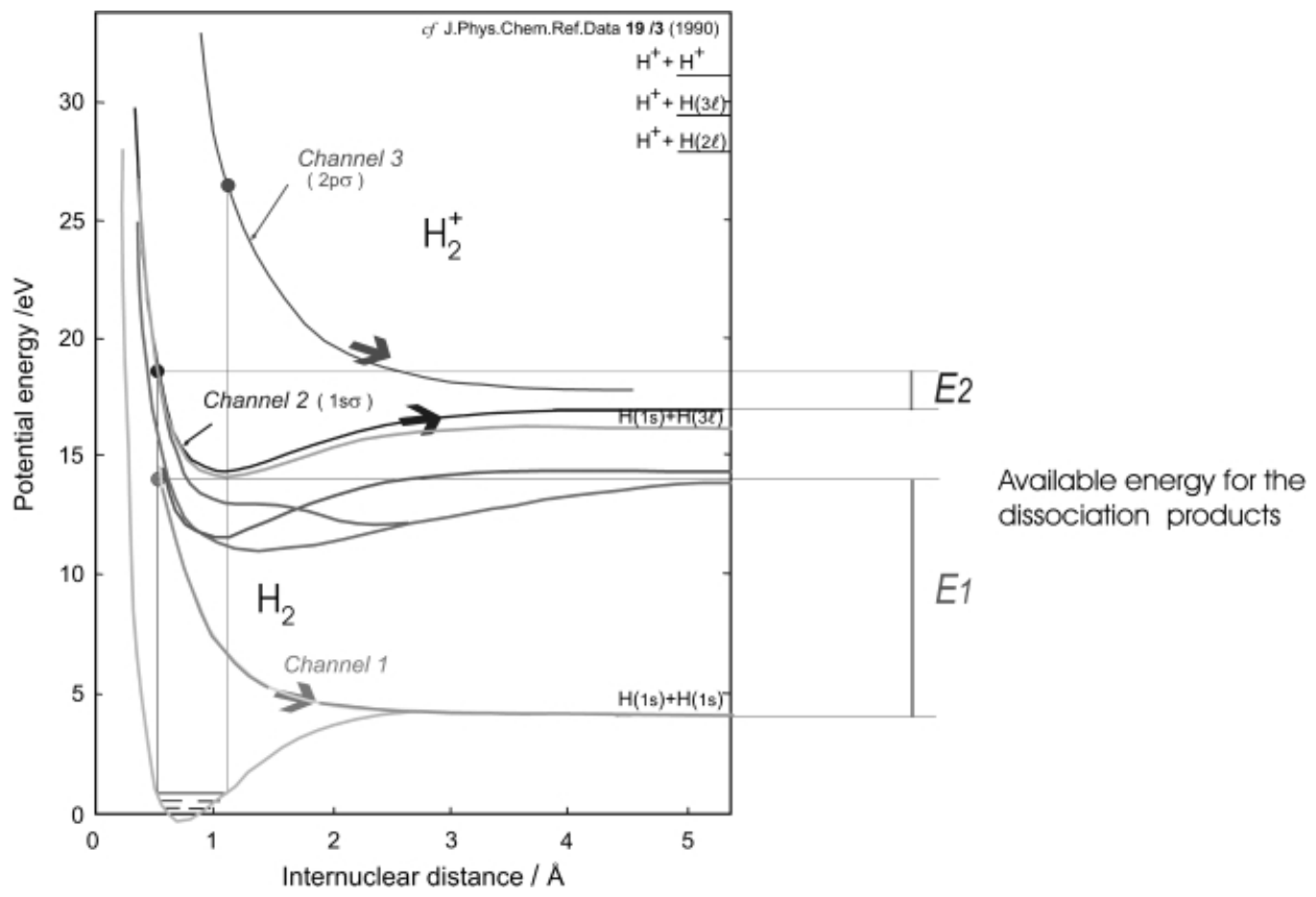

Fig. 10. Illustrative potential curves of the hydrogen molecule. According to the Franck-Condon principle, the transitions take place along vertical lines. Dissociative excitation along channel 2 gives atoms $[\mathrm{H}(1 \mathrm{~s})+\mathrm{H}(3 \ell)]$ with much lower kinetic energy than from the dissociation along channel 1. The threshold for the second channel amounts to $17 \mathrm{eV}$. 
The most suitable transition for the detection of molecular hydrogen by spectroscopy in the visible range is the so-called Fulcher band transition at $\sim 600 \mathrm{~nm}$. In contrast to the electronic transitions of atoms, the detection of a single emission line of a molecular transition is not sufficient to obtain the intensity of an electronic transition, but on the other hand, the Fulcher band transition contains several hundred lines. By chance, the rotational and vibrational populations, which have to be taken into account for the analysis anyway, may help in effectively reducing the number of transitions that ought to be observed. Reference 37 demonstrates that the observation of the first diagonal vibrational transition of the Fulcher band is sufficient to determine the total flux of hydrogen molecules in the parameter range of the TEXTOR edge plasma. ${ }^{38}$ Figure 11 a shows this first diagonal transition - the strongest part of the spectrum of the Fulcher band of $\mathrm{D}_{2}$. The corresponding relative rotational population in the upper electronic state is depicted in Fig. 11b. The population represents a Boltzmann distribution for the observed first eight lines. The spectrum is representative for molecular deuterium in front of the different types of graphite limiters used in TEXTOR (Ref. 38).
The molecular particle flux $\Gamma_{\mathrm{D} 2}$ is determined from the Fulcher band photon flux and conversion factors over the accessible range of plasma parameters in the TEXTOR boundary. The factors for the transfer of photons to molecular fluxes - the so-called $D / X B$ values - have been both measured in calibration experiments with gas puff 37,40 and calculated by a collisional-radiative model. ${ }^{41} \mathrm{Gas}$ puff experiments and modeling provide for an edge electron density of $n_{e}=0.5 \times 10^{18} \mathrm{~m}^{-3}$ and an electron temperature of $T_{e}=50 \mathrm{eV}$, a $D / X B$ value of $\sim 1000$. The $D / X B$ value is not constant but varies with the plasma parameters and amounts, e.g., to 2000 for $n_{e}=5 \times$ $10^{19} \mathrm{~m}^{-3}$ and $T_{e}=40 \mathrm{eV}$.

The measured variations of the molecular flux, i.e., the increase with the edge electron density, is in good agreement with results from the EIRENE modeling. ${ }^{23,32}$ Recombination of deuterium molecules can be neglected thanks to a high edge electron temperature of $>10 \mathrm{eV}$. The Fulcher band spectroscopy thus provides, for the high temperature edge plasma of TEXTOR, the total number of surface-released deuterium molecules-the molecular deuterium flux.

The major questions left are as follows. ${ }^{25,35}$ How much does the molecular flux contribute to the total flux

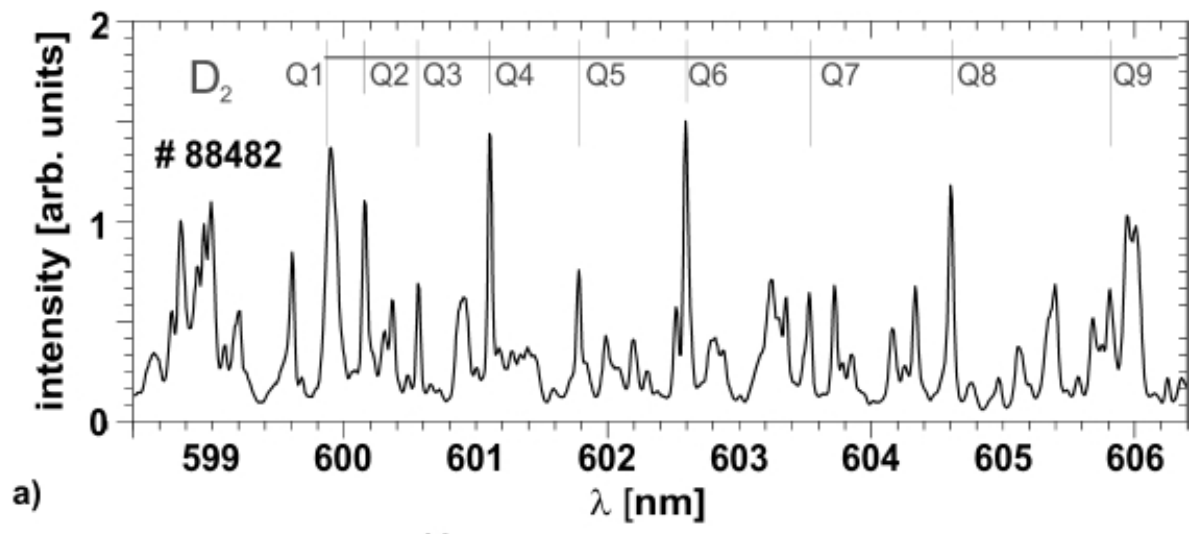

a)

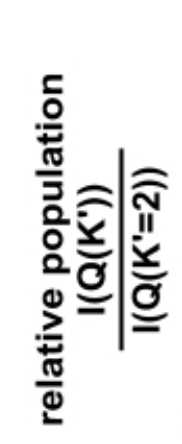

b)

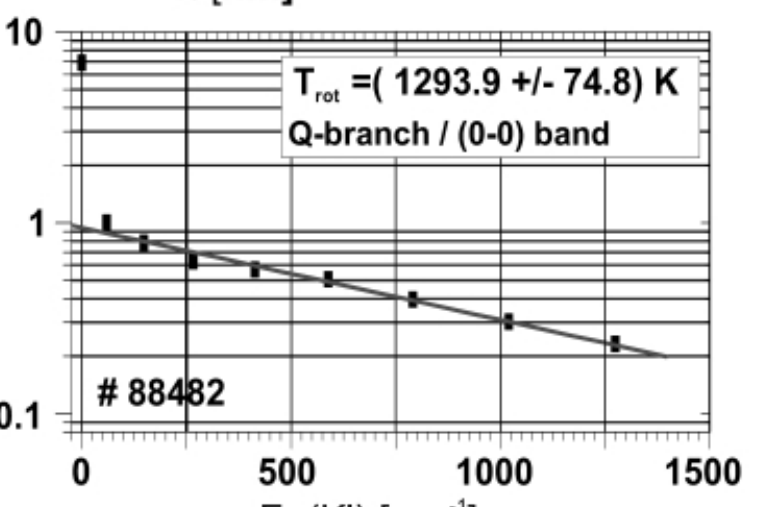

$\mathrm{F}_{\mathrm{v}^{\prime}}\left(\mathrm{K}^{\prime}\right)\left[\mathrm{cm}^{-1}\right]$

Fig. 11. (a) The strongest part of the $\mathrm{D}_{2}$ Fulcher band spectrum. The light has been detected in front of a graphite test limiter in TEXTOR. (b) The corresponding relative rotational population is given in a half-logarithmic Boltzmann plot. The fitted line represents a unique rotational population temperature. ${ }^{39}$ 
of recycled deuterium particles? What is the ratio of molecules to atoms released from the surface? Is there a dependence of type and behavior of the recycled particles on plasma or surface parameters?

A spectroscopic method based on a combination of Fulcher band and Balmer spectroscopy has been developed to determine the recycling hydrogen particle flux..$^{32,42}$ Thereby, it is not sufficient to measure both photon fluxes simultaneously. Some of the atoms measured by means of Balmer spectroscopy might be born at the surface. Others-possibly the major part—are products of the molecular dissociation. A double counting of released particles has to be avoided (see Sec. VI). As a consequence, a plasma-independent variation of the atomicto-molecular ratio was thought to deduce the recycling flux.

Laboratory experiments ${ }^{34}$ predict a change of the surface-released species with variation of the surface temperature-starting with molecules only at low temperatures and ending with atoms at the high end. Experiments with an electrically preheated graphite test limiter in TEXTOR confirmed this change in the release mechanism. ${ }^{43,44}$ Furthermore, these experiments indicate a flux dependence of the threshold value for the direct atomic release. The threshold is shifted to higher surface temperatures in the tokamak. Figure 12a depicts the variation of intensities of different atomic and molecular transitions of hydrogen and deuterium as a function of the graphite surface temperature in a series of identical plasma discharges. The threshold value is $\sim 1100 \mathrm{~K}$. At $1400 \mathrm{~K}$, the direct atomic release dominates.

In Fig. 12b, the measured Balmer-alpha photon flux is plotted against the molecular particle flux for a series of identical plasma discharges but different surface temperatures. Above the threshold, the atomic photon flux increases, and the molecular one decreases. The total flux of recycling particles is given by an extrapolation to the temperature case where molecules are totally absent $(>1750 \mathrm{~K})$. The slope of the curve determines the efficiency factor $\eta$ to $\sim 1$, which means that the average number of atoms that dissociate from a molecule and that potentially emit a Balmer photon is about one. The slope varies only marginally in the accessible plasma parameter range of TEXTOR (Ref. 42), which indicates that the dissociation process remains the same. This leads to consequences in the interpretation of Balmer-alpha line intensities for the determination of the total recycling flux in the case of not-actively heated limiters that are described in Sec. VI.

\section{CONSEQUENCES AND OUTLOOK}

A major consequence of the molecular content in the released hydrogen flux is that neglecting the molecular part when interpreting emission spectroscopy measure- ments can lead to sheer underestimation of the overall fluxes. It is a general practice to convert the photon fluxes measured by emission spectroscopy into particle fluxes by means of so-called inverse photon efficiencies $S /(X B)$, where $S, X$, and $B$ stand for the integral over the rate coefficient for electron-impact ionization, the similar integral but on the excitation rate, and branching ratio of the observed transition, respectively ${ }^{45,46}$ :

$$
\begin{aligned}
\Gamma_{0} & =4 \pi \frac{I_{0}}{h \nu} \frac{\int_{r_{1}}^{r_{2}} n_{0}(r) n_{e}(r)\left\langle\sigma_{i} v_{e}\right\rangle d r}{B \int_{r_{1}}^{r_{2}} n_{0}(r) n_{e}(r)\left\langle\sigma_{e x} v_{e}\right\rangle d r} \\
& =4 \pi \frac{I_{0}}{h \nu} \frac{S}{X B} .
\end{aligned}
$$

A correction to this value for the bare atomic case, as given in most databases ( $\sim 15$ in our case; see e.g., ADAS), was proposed in Ref. 25 to take the molecular contribution properly into account:

$$
(S / X B)_{e f f}=S / X B\left(1+\frac{2 \Gamma_{\mathrm{D} 2}}{\eta \Gamma^{t o t}}\right),
$$

where $\eta$ accounts for the number of emitted photons per molecule ${ }^{\mathrm{b}}$ and $\Gamma_{\mathrm{D} 2} / \Gamma^{t o t}$ for the proportion of molecules in the total flux $\Gamma^{t o t}=\Gamma_{\mathrm{D}}+2 \Gamma_{\mathrm{D} 2}$.

In most cases, the widely used Balmer spectroscopy has been taking a similar correction into account with empirical values, usually $>20$, depending on the local plasma parameters. An overview of $S / X B$ values currently used on different fusion devices is given in Ref. 47.

We propose a correction based on the simultaneous measurement of the molecular flux (typically over the Fulcher band ${ }^{36,48,49}$ ), at least for a few points of the $\left(n_{e}, T_{e}\right)$ parameter space. Careful modeling then gives a recipe, delivering a so-called $D /(X B)$ value for the molecules 44,50 - in analogy to the "atomic" $S /(X B)$ and, finally, the value of $\eta$ to be put into Eq. (4). To quote the value of Sec. V and again give an idea of realistic inverse photon efficiencies, $D / X B$ for a standard TEXTOR case $\left(n_{e}=5 \times 10^{17} \mathrm{~m}^{-3} ; T_{e}=50 \mathrm{eV}\right)$ roughly amounts to 1000 (Ref. 32).

The questions of observed formation of HD molecules and of atomic velocities and penetration depths could recently be addressed more deeply thanks to the highly diagnosed limiter-lock section of TEXTOR. Results

\footnotetext{
${ }^{\mathrm{b}}$ For instance, $\eta$ would be equal to 1 in the case of dissociative excitation with the products $\mathrm{D}^{0}(n=3)+\mathrm{D}^{0}(1 s)$. The "efficiency" quoted in Ref. 25 did not show the precise meaning we attribute to $\eta$ here. Rather, it represented an inverse efficiency, hence, the slight difference in the formulas $(\eta=$ $1 / \eta_{\text {old }}$ ). We now favor the present definition, owing to its straightforward physical meaning as extensively discussed in Ref. 42 [Sec. IV.C, Eq. (16)].
} 

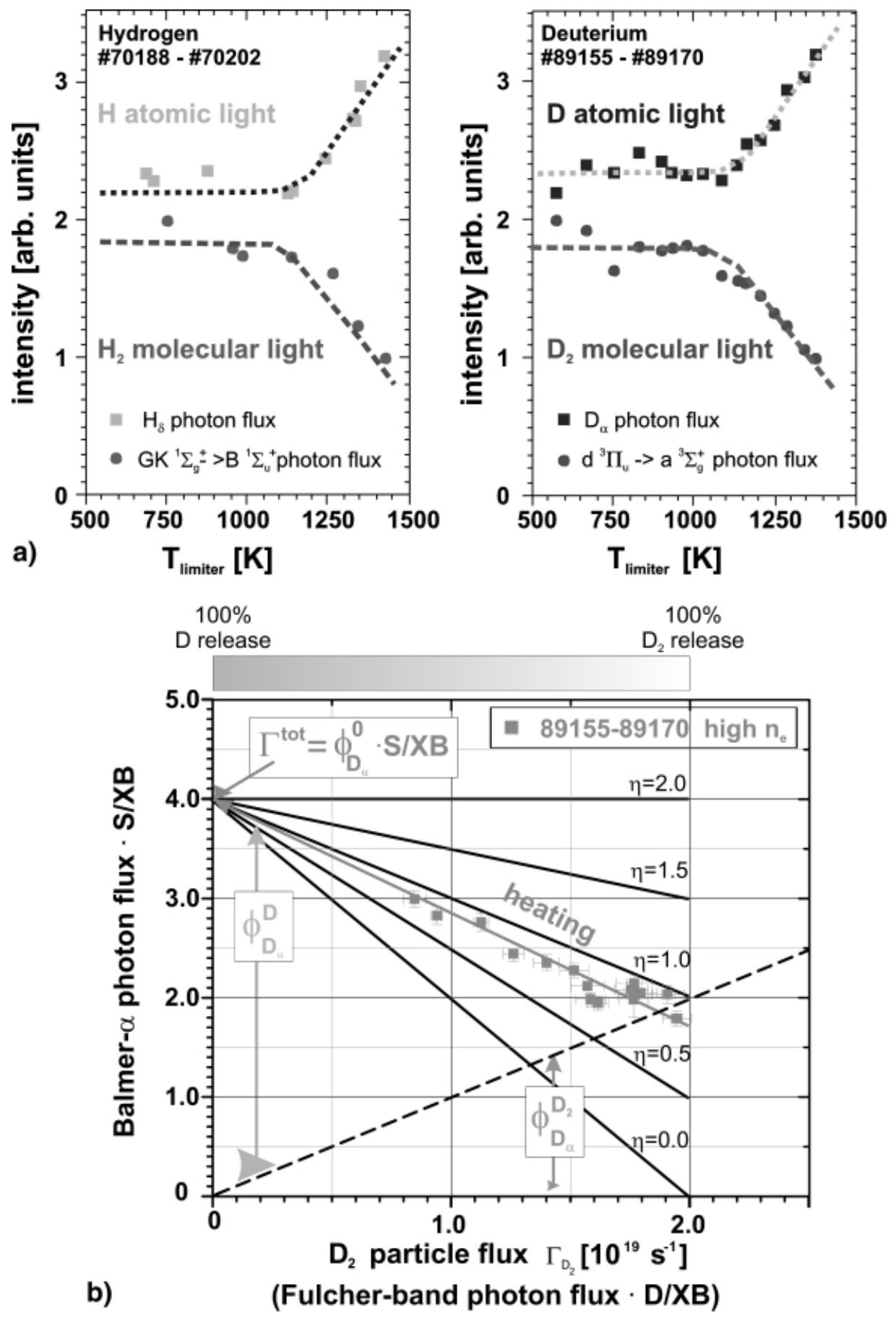

Fig. 12. (a) Variation of the light emission of different atomic and molecular transitions with increase of the graphite surface temperature, ${ }^{36}$ both for hydrogen and deuterium. (b) Above a threshold of $1100 \mathrm{~K}$, the release from the graphite surface changes from molecules to atoms. ${ }^{42}$

are documented in Refs. 51 and 52. These results, which are consequences of the presence of molecular species, are regularly transferred into the EIRENE code for steady improvement.

Ongoing research focuses on the implications of high molecular fluxes; on the influence of surface tempera- ture, which may play a greater role in next-step devices; and on the complex involvement of hydrocarbons in the recycling processes. Most of the results were gained with the simultaneous operation of many different diagnostics, namely, the LIF, emission spectroscopy on atoms with low and high resolution, as well as molecular 
spectroscopy. In addition, recourse to all diagnostics involved in the characterization of the plasma edge, especially to the atomic lithium and helium beams, $38,53,54$ proved to be indispensable.

\section{ACKNOWLEDGMENTS}

The strong support of the TEXTOR team in providing adequate, reproducible tokamak discharges is gratefully acknowledged. The authors are indebted to their colleagues for providing illustration to the present paper, especially to J. D. Hey and A. Pospieszczyk.

\section{REFERENCES}

1. PH. MERTENS, "Sputtering of Limiter and Wall Materials," Proc. 3rd Workshop Plasma and Laser Physics, Ismaïlia, Egypt, p. 141 (1994).

2. B. UNTERBERG et al., Proc. 26th European Physical Society Conf. Controlled Fusion and Plasma Physics, Europhysics Conference Abstracts, 23J, 721 (1999).

3. J. K. EHRENBERG, in Physics of Plasma-Wall Interactions in Controlled Fusion, W. HOFER and J. ROTH, Eds., Academic Press (1996).

4. M. MAYER et al., J. Nucl. Mater., 290-293, 381 (2001).

5. E. TSITRONE et al., Proc. 30th European Physical Society Conf. Controlled Fusion and Plasma Physics, Europhysics Conference Abstracts, 27A, O-2.5A (2003).

6. R. J. GOLDSTON and P. H. RUTHERFORD, Introduction to Plasma Physics, p. 491, Institute of Physics Publishing (2000).

7. G. M. MCCRACKEN and P. E. STOTT, Nucl. Fusion, 19, 889 (1979); see also G. M. MCCRACKEN, Nucl. Fusion, 37, 427 (1997).

8. U. SAMM and TEXTOR TEAM, Plasma Phys. Control. Fusion, 41, B57 (1999).

9. P. C. STANGEBY, The Plasma Boundary of Magnetic Fusion Devices, Institute of Physics Publishing (2000).

10. E. VIETZKE, Contrib. Plasma Phys., 42, 590 (2002).

11. PH. MERTENS and P. BOGEN, Proc. 16th European Physical Society Conf. Controlled Fusion and Plasma Physics, 13B/III, 983 (1989).

12. PH. MERTENS and M. SILZ, J. Nucl. Mater., 241-243, 842 (1997).

13. J. HACKMANN et al., J. Nucl. Mater., 111-112, 221 (1982).

14. K. UCHINO et al., J. Phys. Soc. Jpn., 57, 909 (1988); see also K. MURAOKA et al., J. Nucl. Mater., 176-177, 231 (1990).

15. PH. MERTENS, Proc. 11th Int. Conf. Laser-Aided Plasma Diagnostics, LAPD-11, N. SADEGHI, Ed., Les Houches, France, G8 (2003).

16. T. KAJIWARA et al., Rev. Sci. Instrum., 62, 2345 (1991).

17. K. UCHINO et al., J. Nucl. Mater., 196-198, 210 (1992).

18. R. MAHON et al., IEEE J. Quant. Electron., 15, 441 (1979).

19. PH. MERTENS and P. BOGEN, Appl. Phys. A, 43, 197 (1987).

20. S. BREZINSEK et al., Contrib. Plasma Phys., 42, 657 (2002).

21. SIRAH, Laser- und Plasmatechnik, D-41564 Kaarst, Germany.
22. PH. MERTENS and A. POSPIESZCZYK, J. Nucl. Mater., 266269, 884 (1999).

23. D. REITER et al., "The EIRENE and B2-EIRENE Codes," $F u$ sion Sci. Technol., 47, 172 (2005).

24. B. WELCH et al., Phys. Plasmas, 8, 1253 (2001).

25. PH. MERTENS et al., Plasma Phys. Control. Fusion, 43, A349 (2001).

26. PH. MERTENS, Nachweis von atomarem Wasserstoff und Kohlenstoff im vakuum-ultravioletten Spektralbereich mit einem frequenzverdreifachten Laser, Jül-Report 2154 (1987) (in German).

27. J. D. HEY et al., Contrib. Plasma Phys., 36, 583 (1996).

28. J. D. HEY, C. C. CHU, and PH. MERTENS, Proc. ICSLS 16, AIP Conf. Proc. (2003).

29. A. ESCARGUEL et al., Contrib. Plasma Phys., 42, 622 (2002).

30. J. D. HEY et al., Contrib. Plasma Phys., 42, 635 (2002).

31. J. D. HEY et al., J. Phys. B: At. Mol. Opt. Phys., 37, 2543 (2004).

32. S. BREZINSEK, Untersuchung von atomarem und molekularem Wasserstoff vor einer Graphitoberfläche in einem Hochtemperaturplasma, Jül-Report 3962 (2002) (in German).

33. J. D. HEY et al., J. Phys. B: At. Mol. Opt. Phys., 32, 3555 (1999). 34. P. FRANZEN and E. VIETZKE, J. Vac. Sci. Technol. A, 12, 820 (1994).

35. A. POSPIESZCZYK et al., Contrib. Plasma Phys., 42, 663 (2002). 36. S. BREZINSEK et al., Contrib. Plasma Phys., 42, 668 (2002).

37. S. BREZINSEK et al., J. Nucl. Mater., 313-317, 967 (2003).

38. S. BREZINSEK et al., "Plasma Edge Diagnostics for TEXTOR," Fusion Sci. Technol., 47, 209 (2005).

39. B. UNTERBERG et al., J. Nucl. Mater. (accepted for publication). 40. U. FANTZ et al., J. Nucl. Mater., 313-117, 743 (2003).

41. P. TH. GREENLAND, "The CRMOL Manual: CollisionalRadiative Models for Molecular Hydrogen Plasmas," Jül-Report 3858 (2001).

42. S. BREZINSEK et al., Plasma Phys. Control. Fusion (accepted for publication).

43. A. POSPIESZCZYK, J. Nucl. Mater., 266-269, 138 (1999).

44. S. BREZINSEK et al., Proc. 28th European Physical Society Conf. Controlled Fusion and Plasma Physics, Europhysics Conference Abstracts, $\mathbf{5 . 0 9 3}$ (2001).

45. A. POSPIESZCZYK, in Atomic and Plasma Material Interaction Processes in Controlled Thermonuclear Fusion, p. 213, R. K. JANEV and H. W. DRAWIN, Eds., Elsevier (1993).

46. K. BEHRINGER, J. Nucl. Mater., 145-147, 145 (1987).

47. S. BREZINSEK et al., Phys. Scripta, T111, 42 (2004).

48. U. FANTZ and B. HEGER, Plasma Phys. Control. Fusion, 40, 2023 (1998).

49. B. HEGER et al., J. Nucl. Mater., 290-293, 413 (2001).

50. P. T. GREENLAND, Contrib. Plasma Phys., 42, 608 (2002).

51. S. BREZINSEK et al., Phys. Scripta, T103, 63 (2003).

52. S. BREZINSEK et al., Phys. Scripta, T103, 51 (2003).

53. E. HINTZ and B. SCHWEER, Plasma Phys. Control. Fusion, 37, A87 (1995).

54. B. SCHWEER et al., J. Nucl. Mater., 266-269, 673 (1999). 